\title{
Implicit Function Theorems for Nondifferentiable Mappings ${ }^{1}$
}

\author{
W. BIAN ${ }^{2}$ \\ Communicated by F. A. Potra \\ Published Online: 29 November 2006
}

\begin{abstract}
Sufficient conditions are given for a mapping to be $\gamma-\mathrm{G}$ inverse differentiable. Constrained implicit function theorems for $\gamma-\mathrm{G}$ inverse differentiable mappings are obtained, where the constraint is taken to be either a closed convex cone or a closed subset. A theorem without assuming the $\gamma-\mathrm{G}$ inverse differentiability in a finite-dimensional space is also presented.
\end{abstract}

Key Words. $\gamma-\mathrm{G}$ inverse differentiability, implicit function theorems, Ekeland's variational principle.

\section{Introduction}

This paper is concerned with the concept of $\gamma-\mathrm{G}$ inverse differentiability as defined in Ref. 1 and with implicit function theorems for $\gamma-\mathrm{G}$ inverse differentiable mappings.

It is known that the classical implicit function theorems and open-mapping theorems need the (single-valued) function to be Fréchet differentiable and the derivative to be surjective. Although the Fréchet differentiability can be verified readily in some applications and has received much attention, these results cannot be applied if the function is not Fréchet differentiable or is set-valued. Recently, there have been many publications concerning nonFréchet differentiable problems in which several alternatives to the Fréchet differentiability are used. The first alternative is naturally the Gâteaux differentiability (see Refs. 2-5). The second alternative is to consider set-valued derivatives, including strict prederivatives (Refs. 6 and 7) and high-order set-valued derivatives (Ref. 8). A further alternative is the weak Gâteaux inverse derivative defined by Welsh in Ref. 9 and its general-

\footnotetext{
${ }^{1}$ The author thanks the referees for valuable suggestions concerning the presentation of this paper. He also thanks Professor J. R. L. Webb and Dr. M. French for help.

${ }^{2}$ Research Fellow, School of Electronics and Computer Science, University of Southampton, Southampton, United Kingdom.
} 
ization, the $\gamma-\mathrm{G}$ inverse derivative, given by the authors of Ref. 1 . These concepts are used in Refs. 1, 9 to study the openness of nonlinear operators and in Ref. 1 to analyze the controllability of certain nonlinear systems. The results obtained show that the $\gamma-\mathrm{G}$ inverse differentiability has some potential significance.

In this paper, first we provide new sufficient conditions for a mapping to possess a $\gamma-\mathrm{G}$ inverse derivative (here, we extend the definition to include setvalued mappings). It is shown that many differentiability conditions used for open-mapping or implicit function theorems, including linear approximation and the set-valued derivative, lead to the $\gamma-\mathrm{G}$ inverse differentiability. Then, we apply this differentiability concept and Ekeland's variational principle to consider the implicit function problem for set-valued mappings. The results obtained cannot be derived from the open-mapping theorems obtained in Ref. 1 due to the setting. We present also a theorem in finite-dimensional spaces without using the $\gamma-\mathrm{G}$ inverse differentiability.

\section{2. $\gamma$-Gâteaux Inverse Differentiability}

Given a metric space $X$ with metric $d$, let

$$
B_{X}=\{x \in X: d(x, 0)<1\}
$$

and

$$
B_{X}\left(x_{0}, \alpha\right)=\left\{x \in X: d\left(x, x_{0}\right)<\alpha\right\}, \quad \text { for } x_{0} \in X, \alpha \geq 0 .
$$

If $D, D_{0} \subset X$, let $\bar{D}$ denote the closure of, $D$ let $d\left(x_{0}, D\right)$ denote the distance between $x_{0}$ and $D$, let $H\left(D_{0}, D\right)$ denote the Hausdroff distance between $D$ and $D_{0}$, and let $\chi(D)$ denote the Hausdorff measure of noncompactness of $D$. We recall that $H\left(D_{0}, D\right)$ and $\chi(D)$ are defined respectively as (see Ref. 10)

$$
\begin{aligned}
& H\left(D_{0}, D\right)=\max \left\{\sup _{x \in D} d\left(x, D_{0}\right), \sup _{x \in D_{0}} d(x, D)\right\}, \\
& \chi(D)=\inf \left\{\varepsilon>0: D \subset \cup_{i=1}^{n} B_{X}\left(x_{i}, \varepsilon\right), n>0, x_{1}, \ldots, x_{n} \in D\right\} .
\end{aligned}
$$

For two normed spaces $X, Y$, we let $\mathbb{L}(X, Y)$ denote the space of all bounded linear operators from $X$ to $Y$ endowed with the operator norm.

Definition 2.1. Let $X, Y$ be normed spaces, let $F: \operatorname{Dom}(F) \subset X \rightarrow 2^{Y}$ be a set-valued mapping, and let $x_{0} \in \operatorname{Dom}(F), \gamma \geq 0$. We say that $F$ possesses a $\gamma$ Gâteaux inverse derivative $\Gamma\left(x_{0}\right): Y \rightarrow X$ at $x_{0}$ if, for every $y \in Y$ and sufficiently small $h>0$ with $x_{0}+h \Gamma\left(x_{0}\right) y \in \operatorname{Dom}(F)$, we have

$$
F\left(x_{0}+h \Gamma\left(x_{0}\right) y\right)+h \gamma\|y\| \bar{B}_{Y} \supset F\left(x_{0}\right)+h y+o(h) .
$$

Here, $o(\cdot):(0, \infty) \mapsto Y$ is a single-valued mapping and, $\|o(h)\| / h \rightarrow 0$. as $h \rightarrow$ 0 . $\Gamma\left(x_{0}\right)$ is said to be the $\gamma-\mathrm{G}$ inverse derivative of $F$ at $x_{0}$. 
This notion was introduced first in Ref. 1 for single-valued functions, as a relaxation of the Gâteaux inverse differentiability of Welsh (Ref. 9) and therefore as a relaxation of the invertibility of the Gâteaux derivative. In this section, we will concentrate on giving sufficient conditions for a mapping to be $\gamma$-G inverse differentiable.

We are usually interested in the case where $\gamma<1$, but such an assumption will be not made here explicitly.

Consider the case when the function is Gâteaux (or Fréchet) differentiable and the derivative is locally or globally invertible, as is often required in studying openmapping and implicit function theorems. Then, the 0-Gâteaux inverse derivative is the inverse of the Gâteaux derivative and the local or global invertibility of the derivative implies that the inverse of the derivative is bounded in a certain sense. So, the $\gamma$-G inverse derivative should satisfy a boundedness condition to make it applicable.

Recall that a mapping $f$ (say) is said to be positively homogeneous if $f(k x)-k f(x), \quad$ for all $x \in \operatorname{Dom}(f), k>0$.

Proposition 2.1. Let $X, Y$ be normed spaces, let $P \subset X$ be a cone, let $L: P \rightarrow Y$ be a positively homogeneous operator, and let $c>0, \gamma \geq 0$ be such that

$$
B_{Y} \subset L\left(P \cap c B_{X}\right)+\gamma B_{Y} .
$$

Suppose that $F: D \rightarrow 2^{Y}$ is a set-valued mapping with $D=\operatorname{Dom}(F) \subset X$ and that $\alpha \geq 0, x_{0} \in P \cap D, \varepsilon>0$ are such that

$$
F\left(x_{0}\right) \subset F(x)-L\left(x-x_{0}\right)+\alpha\left\|x-x_{0}\right\| \bar{B}_{Y}, \quad \text { for all } x \in D \cap B_{X}\left(x_{0}, \varepsilon_{0}\right) .
$$

Then, for each $\lambda>1, F$ has a $\lambda(\gamma+\alpha c)-G$ inverse derivative $\Gamma\left(x_{0}\right)$ at $x_{0}$ such that

$$
\Gamma\left(x_{0}\right) B_{Y} \subset P \quad \text { and } \quad\left\|\Gamma\left(x_{0}\right) y\right\| \leq \lambda c\|y\|, \quad \text { for all } y \in Y .
$$

In addition, if $P$ is convex, then condition (3) can be replaced by $F\left(x_{0}\right) \subset F(x)-L\left(x-x_{0}\right)+\alpha\left\|x-x_{0}\right\| \bar{B}_{Y}, \quad$ for all $x \in P \cap D \cap B_{X}\left(x_{0}, \varepsilon_{0}\right)$.

Proof. Let $\lambda>1$ be given. Then, for each $y \in Y$, (2) implies that there exist $u_{y}=u(\lambda, y) \in P \cap B_{X}$ and $v_{y}=v(\lambda, y) \in B_{Y}$ such that

$$
y=\lambda\|y\| L\left(c u_{y}\right)+\lambda\|y\| \gamma v_{y}=\lambda c\|y\| L\left(u_{y}\right)+\lambda \gamma\|y\| v_{y} .
$$


For each $y \in Y$, let

$$
\Gamma\left(x_{0}\right) y=\lambda c\|y\| u_{y},
$$

where $u_{y} \in P \cap B_{X}$ satisfies (6); in the case where there are several such points, we fix one so that $\Gamma\left(x_{0}\right)$ is a well-defined mapping from $Y$ to $X$. Since $u_{y} \in P \cap B_{X}$ and $P$ is a cone, we see that (4) is satisfied.

Suppose that $y \in Y$ and $h>0$ is so small that

$$
x_{0}+h \Gamma\left(x_{0}\right) y \in D \cap B_{X}\left(x_{0}, \varepsilon_{0}\right) \text {. }
$$

From (6) and the homogeneity of $L$, it follows that

$$
L\left(\Gamma\left(x_{0}\right) y\right)=\lambda c\|y\| L\left(u_{y}\right)=y-\lambda \gamma\|y\| v_{y} .
$$

So, by (3), we have

$$
\begin{aligned}
F\left(x_{0}\right)+h y & =F\left(x_{0}\right)+L\left(h \Gamma\left(x_{0}\right) y\right)+h \lambda \gamma\|y\| v_{y} \\
& \subset F\left(x_{0}+h \Gamma\left(x_{0}\right) y\right)+h \alpha\left\|\Gamma\left(x_{0}\right) y\right\| \bar{B}_{Y}+h \lambda \gamma\|y\| B_{Y} \\
& \subset F\left(x_{0}+h \Gamma\left(x_{0}\right) y\right)+h \lambda(\Gamma+\alpha c)\|y\| \bar{B}_{Y} .
\end{aligned}
$$

Hence, $\Gamma\left(x_{0}\right)$ is a $\lambda(\gamma+\alpha c)-\mathrm{G}$ inverse derivative of $F$ at $x_{0}$.

Now, we suppose that $P$ is a convex cone. Since $x_{0} \in P$ and $\Gamma\left(x_{0}\right)$ is a mapping into $P$, we see $x_{0}+h \Gamma\left(x_{0}\right) y \in P$ for all $y \in Y$ and $h>0$. Consequently, we can use (5), instead of (3), to prove that $\Gamma\left(x_{0}\right)$ is a $\lambda(\gamma+\alpha c)-\mathrm{G}$ inverse derivative of $F$ at $x_{0}$.

In the case where $F$ is single-valued, (3) can be rewritten as

$$
\left\|F(x)-F\left(x_{0}\right)-L\left(x-x_{0}\right)\right\| \leq \alpha\left\|x-x_{0}\right\| .
$$

In addition, if $\alpha$ can be taken to be arbitrarily small, then $L$ will be the linear approximation used in Ref. 10 . Generally, $L$ is an approximation to $F$ with relative error bounded by $\alpha$, so we may call it $\alpha$-approximation. The next result shows that this $\alpha$-approximation could be a subset of bounded linear operators.

Proposition 2.2. $\quad$ Let $X$ be a normed space, let $Y$ be a Banach space, let $P \subset X$ be a closed convex cone, let $\mathbb{L} \subset(X, Y)$ be a bounded convex subset, and let $c>0$ be such that

$$
B_{Y} \subset L\left(P \cap c B_{X}\right), \quad \text { for each } L \in \mathcal{L} .
$$

Suppose that $f: X \rightarrow Y$ is a continuous function and that $x_{0} \in P, \alpha \geq 0, \varepsilon_{0}>0$ are such that

$$
f(x)-f\left(x_{0}\right) \in \mathcal{L}\left(x-x_{0}\right)+\alpha\left\|x-x_{0}\right\| \bar{B}_{Y}, \quad \text { for all } x \in P \cap B_{X}\left(x_{0}, \varepsilon_{0}\right) .
$$


Then, for each $\lambda>1$ and $\gamma>\chi(\mathcal{L}), f$ possesses a $\lambda c(\gamma+\alpha)-G$ inverse derivative $\Gamma\left(x_{0}\right)$ at $x_{0}$ and (4) is satisfied.

Proof. Since $\gamma>\chi(\mathcal{L})$, there exist $L_{1}, \ldots, L_{n} \in \mathcal{L}$ such that $\mathcal{L} \subset \bigcup_{i=1}^{n} B_{\mathbb{L}(X, Y)}\left(L_{i}, \gamma\right)$.

Let

$$
\mathcal{T}=\operatorname{co}\left\{L_{1}, \ldots, L_{n}\right\}
$$

Then, $\mathcal{T} \subset \mathcal{L}$ is a compact convex subset of $\mathbb{L}(X, Y)$ and $f(x)-f\left(x_{0}\right) \in \mathcal{T}\left(x-x_{0}\right)+(\alpha+\gamma)\left\|x-x_{0}\right\| B_{Y}, \quad$ for all $x \in P \cap B_{X}\left(x_{0}, \varepsilon_{0}\right)$.

Let $\lambda>1$ be given and let $y \in Y$. From (7), it follows that, for each $L \in \mathcal{T}$,

$$
R_{y}(L):=\left\{x \in B_{X}(0, \lambda c\|y\|) \cap P: L x-y=0\right\}
$$

is nonempty. It is standard to check that $R_{y}(L)$ is also closed and convex. By Proposition 1.5.1 in Ref. 11, $R_{y}(\cdot)$ is lower semicontinuous as a set-valued mapping. By Lemma 1.2.1 in Ref. 10, there exists a continuous operator $L \mapsto x(L, y) \in R_{y}(L)$ that is,

$$
y=L x(L, y), \quad x(L, y) \in P, \quad\|x(L, y)\| \leq \lambda c\|y\| .
$$

Since $P$ is a cone, there exists $k>0$ such that

$$
x_{0}+h x(L, y) \in P \cap B_{X}\left(x_{0}, \varepsilon_{0}\right), \quad \text { for each } h \in[0, k] \text { and each } L \in \mathcal{T} .
$$

By our assumptions, there exists $S \in \mathcal{T}$ such that

$$
\begin{array}{r}
\left\|f\left(x_{0}+h x(L, y)\right)-f\left(x_{0}\right)-h \operatorname{Sx}(L, y)\right\| \leq(\alpha+\gamma)\|h x(L, y)\|, \\
\text { for all } h \in[0, k] .
\end{array}
$$

Let

$$
\Phi(L)=\{S \in \mathcal{T}: S \text { satisfies (10) }\}
$$

From the compactness of $\mathcal{T}$ and (9), it follows that $\Phi$ is a well defined and compact set-valued mapping on $\mathcal{T}$. To prove that $\Phi$ is upper semicontinuous, we suppose that $D \subset \mathbb{L}(X, Y)$ is a closed subset and $L_{n} \in \Phi^{-1}(D)$ with $L_{n} \rightarrow L$. Then, there exists $S_{n} \in \mathcal{T} \cap D$ such that

$$
\begin{array}{r}
\left.\left\|f\left(x_{0}+h x\left(L_{n}, y\right)\right)-f\left(x_{0}\right)-h S_{n} x\left(L_{n}, y\right)\right\| \leq(\alpha+\gamma)\left\|h x\left(L_{n}, y\right)\right\|\right\}, \\
\text { for all } h \in[0, k] .
\end{array}
$$


We may suppose that $S_{n} \rightarrow S \in \mathcal{T} \cap D$, due to the compactness of $\mathcal{T}$ and closedness of $D$. Since $L \mapsto x(L, y)$ is continuous, we have

$$
\begin{array}{r}
\left.\left\|f\left(x_{0}+h x(L, y)\right)-f\left(x_{0}\right)-h S_{n} x(L, y)\right\| \leq(\alpha+\gamma)\|h x(L, y)\|\right\}, \\
\text { for all } h \in[0, k] .
\end{array}
$$

which implies that $L \in \Phi^{-1}(D)$. So, $\Phi^{-1}(D)$ is closed; therefore, $\Phi$ is upper semicontinuous. Applying the Kakutani fixed-point theorem (Ref. 10, Theorem 24.4), we see that there exists $L_{y} \in \mathcal{T}$ such that $L_{y} \in \Phi\left(L_{y}\right)$, that is,

$$
\begin{aligned}
& \left\|f\left(x_{0}+h x\left(L_{y}, y\right)\right)-f\left(x_{0}\right)-h L_{y} x\left(L_{y}, y\right)\right\| \\
& \leq(\alpha+\gamma)\left\|h x\left(L_{y}, y\right)\right\| \leq \lambda c(\alpha+\gamma) h\|y\|,
\end{aligned}
$$

for all $h \in[0, k]$ Since $L_{y} x\left(L_{y}, y\right)=y$, there exists $w_{y} \in B_{Y}$ such that

$$
f\left(x_{0}+h x\left(L_{y}, y\right)\right)=f\left(x_{0}\right)+h y+h \lambda c(\alpha+\gamma)\|y\| w_{y} .
$$

For each $y \in Y$ there may be several $x\left(L_{y}, y\right)$ with $x(\cdot, \cdot)$ and $L_{y}$ as above; we fix one arbitrarily so that $y \mapsto \Gamma\left(x_{0}\right) y:=x\left(L_{y}, y\right)$ is a well-defined mapping from $Y$ to $X$. From (11), we see that $\Gamma\left(x_{0}\right)$ is a $\lambda c(\alpha+\gamma)-\mathrm{G}$ inverse derivative of $f$ at $x_{0}$. Obviously, (4) is satisfied. This completes the proof.

Remark 2.1. If $f$ has a strict prederivative $\mathcal{A} \in \mathbb{L}(X, Y)$ at $x_{0}$, that is,

$$
f\left(x_{1}\right) \in f\left(x_{2}\right)+\mathcal{A}\left(x_{1}-x_{2}\right)+\varepsilon\left\|x_{1}-x_{2}\right\| B_{Y}, \quad \text { for all } x_{1}, x_{2} \in \operatorname{Dom}(f),
$$

then (8) is satisfied. This strict prederivative assumption was used in Ref. 7 together with condition (7) for implicit function problems, where $P=X$.

Proposition 2.3. Let $X, Y$ be normed spaces and let $P \subset X$ be a cone. Suppose that $f: \operatorname{Dom}(f) \subset X \rightarrow Y$ is a single-valued function which is Gâteaux differentiable at $x_{0}$ with Gateaux derivative $\mathrm{D} f\left(x_{0}\right)$. Suppose that there exist $c>0, \gamma \geq 0$ such that

$$
B_{Y} \subset \mathrm{D} f\left(x_{0}\right)\left(P \cap c B_{X}\right)+\gamma B_{Y} .
$$

Then, for each $\lambda>1, f$ possesses a $\lambda \gamma-G$ inverse derivative $\Gamma\left(x_{0}\right)$ at $x_{0}$ satisfying (4).

Proof. Let $\lambda>1$ be given. From (12), it follows that, for each $y \in Y$, there exists $v_{y} \in B_{X} \cap P, w_{y} \in B_{Y}$ such that

$$
y=\mathrm{D} f\left(x_{0}\right)\left(c \lambda\|y\| v_{y}\right)+\gamma \lambda\|y\| w_{y} .
$$

Let

$$
\Gamma\left(x_{0}\right) y=c \lambda\|y\| v_{y},
$$


where for each $y \in Y$, we fix a point $v_{y}$ in $B_{X} \cap P$ satisfying (13) so that $\Gamma\left(x_{0}\right)$ is a well-defined operator from $Y$ to $X$. By the definition of D $f\left(x_{0}\right)$ and (13), we see that

$$
f\left(x_{0}+h \Gamma\left(x_{0}\right) y\right)=f\left(x_{0}\right)+h y+h \lambda \gamma\|y\|\left(-w_{y}\right)+o(h) ;
$$

that is, $\Gamma\left(x_{0}\right)$ is a $\lambda \gamma-\mathrm{G}$ inverse derivative of $f$ at $x_{0}$. Since $v_{y} \in B_{x},(4)$ is satisfied.

\section{Implicit Function Theorems}

In this section, we use the concept of $\gamma-\mathrm{G}$ inverse differentiability to derive some constrained implicit function theorems. We remark that open-mapping theorems were obtained in Ref. 1 in terms of $\gamma-\mathrm{G}$ inverse differentiability. Although open-mapping theorems and implicit function theorems are often equivalent to each other, the results presented below cannot be derived from those of Ref. 1, even when the mappings involved are all single-valued.

The mappings considered here are of the form $F(x, u)+G(x, u)$, with both $F$ and $G$ set-valued. The $\gamma-G$ inverse differentiability assumption is imposed only on the mapping $x \mapsto F(x, u)$, while $x \mapsto G(x, u)$ is assumed to be locally Lipschitz; that is, there exists $l>0$ and a neighborhood $\mathcal{U}$ of each $x$ such that

$$
G\left(x_{1}, u\right) \subset G\left(x_{2}, u\right)+l d\left(x_{1}, x_{2}\right) \bar{B}, \quad \text { for all } x_{1}, x_{2} \in \mathcal{U} .
$$

We recall (Ref. 11) that a mapping $\mathcal{F}$ is said to be pseudo-Lipschitz around $(x, y) \in$ $\operatorname{Graph}(\mathcal{F})$, the graph of $\mathcal{F}$, with constant $l$ if there exist neighborhoods $\mathcal{U}$ of $x$ and $\mathcal{V}$ of $y$ such that

$$
\mathcal{F}\left(x_{1}\right) \cap \mathcal{V} \subset \mathcal{F}\left(x_{2}\right)++l d\left(x_{1}, x_{2}\right) \bar{B}_{z}, \quad \text { for all } x_{1}, x_{2} \in \mathcal{U}
$$

We shall be imposing the following conditions.

(H1) $X, Y$ are Banach spaces, $U$ is a metric space, and $K \subset \Omega \subset X$.

(H2) $F, G: \bar{\Omega} \times U \rightarrow 2^{Y}$ are two set-valued mappings with bounded and closed values, and $x_{0} \in K, u_{0} \in U$ are such that

$$
\lim _{u \rightarrow u_{0}} d\left(0, F\left(x_{0}, u\right)+G\left(x_{0}, u\right)\right)=0 .
$$

(H3) For each $u \in B_{U}\left(u_{0}, r\right), x \mapsto G(x, u)$ is $\varepsilon$ - $\delta$-usc and Lipschitz with constant $k$ when restricted to $K \cap B_{X}\left(x_{0}, \delta\right)$, where $r, \delta>0, k \geq 0$ are given.

We consider first the case when the mapping $x \mapsto F(x, u)$ is only closed or is $\varepsilon$ - $\delta$-usc and the constraint is a closed convex cone. The same problem with noncone constraint will be considered later. 
Theorem 3.1. Let $\Omega=K$ be a closed convex cone. Suppose that $F, G$ satisfy (H1)-(H3) and (F1)-(F2) below:

(F1) For each $u \in B_{U}\left(u_{0}, r\right), x \mapsto F(x, u)$ is either a closed single-valued mapping or an $\varepsilon-\delta$-usc set-valued mapping.

(F2) For each $u \in B_{U}\left(u_{0}, r\right), x \mapsto F(x, u)$ possesses a $\gamma-G$ inverse derivative $\Gamma_{u}(x)$ at each $x \in K \cap B_{X}\left(x_{0}, \delta\right)$ such that

$$
\begin{aligned}
& \left\|\Gamma_{u}(x) y\right\| \leq M\|y\|, \quad \text { for all } y \in B_{Y}, x \in K \cap B_{X}\left(x_{0}, \delta\right) \\
& \Gamma_{u}(x) B_{Y} \subset K, \quad \text { for all } x \in K \cap B_{X}\left(x_{0}, \delta\right), \\
& \text { where } \gamma \geq 0, M>0, \text { and } \gamma+k M<1
\end{aligned}
$$

Let

$$
\begin{aligned}
\eta= & \sup \left\{r_{1} \in(0, r]: \sup _{u \in B_{U}\left(u_{0}, r_{1}\right)} d\left(0, F\left(x_{0}, u\right)\right.\right. \\
& \left.\left.+G\left(x_{0}, u\right)\right)<\left[\frac{(1-\gamma-k M)}{M}\right] \delta\right\} .
\end{aligned}
$$

Then, for each $u \in B_{U}\left(u_{0}, \eta\right)$, there exists $x_{u} \in K \cap B_{X}\left(x_{0}, \delta\right)$ such that

$$
0 \in F\left(x_{u}, u\right)+G\left(x_{u}, u\right) \text { and } \lim _{u \rightarrow u_{0}} x_{u}=x_{0} .
$$

In addition, if $u \mapsto F(x, u)$ is locally Lipschitz at $u_{0}$ with constant $l$ uniformly in $x \in K \cap B_{X}\left(x_{0}, \delta\right)$, then the (constrained) implicit mapping

$$
W(u):=\{x \in K: 0 \in F(x, u)+G(x, u)\}
$$

is pseudo-Lipschitz around $\left(u_{0}, x_{0}\right)$ with constant $l M /(1-\gamma-k M)$.

Proof. From (14), it follows that the number $\eta$ given in (17) is well defined. Let $u \in B_{U}\left(u_{0}, \eta\right)$ be given. Then, there exists $\varepsilon_{0} \in(0,(1-\gamma-k M) / M)$ such that

$$
d\left(0, F\left(x_{0}, u\right)+G\left(x_{0}, u\right)\right)<\varepsilon_{0} \delta .
$$

Define a new metric $d_{0}$ on $K$ by

$$
d_{0}\left(x_{1}, x_{2}\right)=\max \left\{\left\|x_{1}-x_{2}\right\|, \theta\left\|F\left(x_{1}, u\right)-F\left(x_{2}, u\right)\right\|\right\}, \quad \text { for } x_{1}, x_{2} \in K .
$$

If $x \mapsto F(x, u)$ is a closed single-valued mapping, then $\theta>0$ is taken to satisfy $\theta(1+\gamma)<M$; if $x \mapsto F(x, u)$ is an $\varepsilon$ - $\delta$-usc set-valued mapping, then $\theta=0$. By (F1), the space $K$ endowed with the metric $d_{\theta}$ is complete and is denoted 
by $K_{\theta}$ in the following. From our assumptions, it follows that $F(\cdot, u)+G(\cdot, u)$ is $\varepsilon$ - $\delta$-usc from $K_{\theta}$ to $Y$; therefore, the function

$$
V(x):=d(0, F(x, u)+G(x, u))
$$

is lower semicontinuous (see Ref. 12). For a given $\varepsilon \in\left(\varepsilon_{0}(1-\gamma-k M) / M\right)$, by applying the Ekeland variational principle (Theorem 3.3.1 in Ref. 11) to the function $V(x)$ in the space $K_{\theta}$, we obtain $x_{u} \in K_{0}$ such that

$$
\begin{aligned}
& V\left(x_{u}\right) \leq V(x)+\varepsilon d_{\theta}\left(x, x_{u}\right), \quad \text { for all } x \in K_{\theta}, \\
& d_{\theta}\left(x_{u}, x_{0}\right) \leq\left[V\left(x_{0}\right)-V\left(x_{u}\right)\right] / \varepsilon .
\end{aligned}
$$

(18) and (20) imply that

$$
\left\|x_{u}-x_{0}\right\| \leq d_{\theta}\left(x_{u}, x_{0}\right) \leq \varepsilon_{0} \delta / \varepsilon<\delta ;
$$

therefore,

$x_{u} \in K \cap B_{x}\left(x_{0}, \delta\right)$.

Next, we prove that

$$
0 \in F\left(x_{0}, u\right)+G\left(x_{u}, u\right) .
$$

If this claim is not true, then

$$
a:=d\left(0, F\left(x_{u}, u\right)+G\left(x_{u}, u\right)\right)>0 .
$$

Let $\beta>0$ be so small that

$$
k M+\varepsilon M+\gamma+\beta<1 .
$$

For each $h>0$, by the definition of $d\left(0, F\left(x_{u}, u\right)+G\left(x_{u}, u\right)\right)$, there exists $y:=$ $y(h) \in F\left(x_{u}, u\right)+G\left(x_{u}, u\right), y \neq 0$, such that

$$
\|y\| \leq d\left(0, F\left(x_{u}, u\right)+G\left(x_{u}, u\right)\right)+h \beta=V\left(x_{u}\right)+h \beta .
$$

By (16),

$$
z=\Gamma_{u}\left(x_{u}\right)(-y / \lambda\|y\|), \quad \text { with } \lambda>1,
$$

is well defined and

$$
z \in K,\|z\| \leq M / \lambda
$$

Since $K$ is a convex cone, $x_{u}+h z \in K$. Replacing $x$ in (19) by $x_{u}+h z$, we obtain

$$
\begin{aligned}
V\left(x_{u}\right) \leq & V\left(x_{u}+h z\right)+\varepsilon d_{\theta}\left(x_{u}+h z, x_{u}\right) \\
\leq & d\left(0, F\left(x_{u}+h z, u\right)+G\left(x_{u}, u\right)\right)+H_{Y}\left(G\left(x_{u}+h z, u\right), G\left(x_{u}, u\right)\right) \\
& +\varepsilon \max \left\{h\|z\|, \theta\left\|F\left(x_{u}+h z, u\right)-F\left(x_{u}, u\right)\right\|\right\} \\
\leq & d\left(0, F\left(x_{u}+h z, u\right)+h(\gamma / \lambda) \bar{B}_{Y}+G\left(x_{u}, u\right)\right)+H_{Y}\left(\{0\}, h(\gamma / \lambda) \bar{B}_{Y}\right)
\end{aligned}
$$




$$
\begin{aligned}
& +H_{Y}\left(G\left(x_{u}+h z, u\right), G\left(x_{u}, u\right)\right) \\
& +\varepsilon \max \left\{h\|z\|, \theta\left\|F\left(x_{u}+h z, u\right)-F\left(x_{u}, u\right)\right\|\right\} .
\end{aligned}
$$

Here, $H_{Y}(\cdot, \cdot)$ denotes the Hausdorff distance on $Y$. By Definition 2.1, we have

$$
F\left(x_{u}+h z, u\right)+h(\gamma / \lambda) B_{Y} \supset F\left(x_{u}, u\right)+h(-y / \lambda\|y\|)+o(h) .
$$

Suppose that $h$ is small enough so that $h<\lambda a$ (so $h<\lambda\|y\|)$ and $x_{u}+h z \in$ $B_{x}\left(x_{0}, \delta\right)$. As $G$ is Lipschitz in $x, \lambda>1$ and $y \in F\left(x_{u}, u\right)+G\left(x_{u}, u\right)$, from (21) it follows that

$$
\begin{aligned}
V\left(x_{u}\right) \leq & d\left(0, F\left(x_{u}, u\right)-h y / \lambda\|y\|+o(h)+G\left(x_{u}, u\right)\right)+h \gamma \\
& +k h\|z\|+\varepsilon h \max \left\{M / \lambda, \theta+\theta\|o(h)\| / h+\theta_{\gamma}\right\} \\
\leq & \|(1-h / \lambda\|y\|) y\|+\|o(h)\|+h \gamma+k h M / \lambda \\
& +\varepsilon h \max \left\{M / \lambda, \theta+\theta\|o(h)\| / h+\theta_{\gamma}\right\} .
\end{aligned}
$$

So,

$$
\begin{gathered}
V\left(x_{u}\right) \leq V\left(x_{u}\right)+h \beta-h / \lambda+\|o(h)\|+h \gamma+k h M / \lambda \\
+\varepsilon h \max \left\{M / \lambda, \theta+\theta\|o(h)\| / h+\theta_{\gamma}\right\} ;
\end{gathered}
$$

that is,

$$
1 / \lambda \leq \beta+\|o(h)\| h+k M / \lambda+\gamma+\varepsilon \max \left\{M / \lambda, \theta+\theta\|o(h)\| / h+\theta_{\gamma}\right\} .
$$

By letting $\lambda \rightarrow 1, h \rightarrow 0$, we have

$$
\begin{aligned}
1 & \leq \beta+k M+\gamma+\varepsilon \max \{M, \theta(1+\gamma)\} \\
& =\beta+k M+\gamma+k M+\gamma+\varepsilon M<1,
\end{aligned}
$$

which is a contradiction. Therefore,

$$
0 \in F\left(x_{u}, u\right)+G\left(x_{u}, u\right) .
$$

From (14) and (20), we see also that

$$
\begin{aligned}
\left\|x_{u}-x_{0}\right\| & \leq d_{\theta}\left(x_{u}, x_{0}\right) \\
& \leq(1 / \varepsilon) d\left(0, F\left(x_{0}, u\right)+G\left(x_{0}, u\right)\right) \rightarrow 0, \text { as } u \rightarrow u_{0} .
\end{aligned}
$$

In addition, if $u \mapsto F(x, u)+G(x, u)$ is locally Lipschitz at $u_{0}$ with constant $l>0$, then $u \mapsto F\left(x_{0}, u\right)+G\left(x_{0}, u\right)$ is $\varepsilon$ - $\delta$-usc near $u_{0}$ and therefore is closed when restricted to a closed neighborhood of $u_{0}$. So, (14) implies $\left(u_{0}, x_{0}\right) \in$ $\operatorname{Graph}(W)$. We fix a number $r_{1} \in(0, \eta)$. Then,

$$
\sup _{u \in B_{U}\left(u_{0}, r_{1}\right)} d\left(0, F\left(x_{0}, u\right)+G\left(x_{0}, u\right)\right)<[(1-\gamma-k M) / M] \delta ;
$$

therefore, there exists $\varepsilon_{0} \in(0,(1-\gamma-k M) / M)$ such that

$$
d\left(0, F\left(x_{0}, u\right)+G\left(x_{0}, u\right)\right)<\varepsilon_{0} \delta, \quad \text { for all } u \in B_{U}\left(u_{0}, r_{1}\right) .
$$


Let $r_{2}>0$ be small enough such that $u \mapsto F(x, u)+G(x, u)$ is Lipschitz with constant $l$ when restricted to $B_{U}\left(u_{0}, r_{2}\right)$ and $r_{2}<\min \left\{\delta \varepsilon_{0} / \Lambda l, r_{1}\right\}$. Let $d_{U}$ denote the metric of the space $U$. For all given $u, u_{1} \in B_{U}\left(u_{0}, r_{2}\right)$, we claim that

$$
W\left(u_{1}\right) \cap B_{X}\left(x_{0}, \delta / 2\right) \subset W(u)+[l M /(1-\gamma-k M)] d_{U}\left(u, u_{1}\right) \bar{B}_{X} .
$$

In fact, for a given $\bar{x} \in W\left(u_{1}\right) \cap B_{X}\left(x_{0},(1 / 2) \delta\right)$ and a given $\varepsilon \in\left(\varepsilon_{0},(1-\right.$ $\gamma-k M) / M)$, by applying the Ekeland variational principle, we see that there exists $\hat{x} \in K$ satisfying (19) with the same $\theta$ and

$$
\|\hat{x}-\bar{x}\| \leq d_{\theta}(\hat{x}, \bar{x}) \leq(1 / \varepsilon) d(0, F(\bar{x}, u)+G(\bar{x}, u)) .
$$

Noting that $0 \in F\left(\bar{x}, u_{1}\right)+G(\bar{x}, u)$, we have

$$
\begin{aligned}
\|\hat{x}-\bar{x}\| & \leq(1 / \varepsilon) H_{Y}\left(F(\bar{x}, u)+G(\bar{x}, u), F\left(\bar{x}, u_{1}\right)+G\left(\bar{x}, u_{1}\right)\right) \\
& \leq l d_{U}\left(u, u_{1}\right) / \varepsilon \\
& <2 l r_{2} / \varepsilon \\
\left\|\hat{x}-x_{0}\right\| & \leq\|\hat{x}-\bar{x}\|+\left\|\bar{x}-x_{0}\right\| \\
& \leq 2 l r_{2} / \varepsilon+\delta / 2<\delta .
\end{aligned}
$$

Hence, $\hat{x} \in B_{X}\left(x_{0}, \delta\right)$; therefore, $0 \in F(\hat{x}, u)+G(\hat{x}, u)=0$ or $\hat{x} \in W(u)$ as shown above. Moreover, from (23), it follows that

$$
d(\bar{x}, W(u)) \leq\|\bar{x}-\hat{x}\| \leq(l / \varepsilon) d_{U}\left(u, u_{1}\right)
$$

or

$$
\bar{x} \in W(u)+(l / \varepsilon) d_{U}\left(u, u_{1}\right) \bar{B}_{X} .
$$

Since $\bar{x} \in W\left(u_{1}\right) \cap B_{X}\left(x_{0}, \delta / 2\right)$ is arbitrary, we see that

$$
W\left(u_{1}\right) \cap B_{X}\left(x_{0}, \delta / 2\right) \subset W(u)+(l / \varepsilon) d_{U}\left(u, u_{1}\right) \bar{B}_{X} .
$$

Since $\varepsilon \in\left(\varepsilon_{0},(1-\gamma-k M) / M\right)$ is arbitrary, it can be replaced by $(1-\gamma-$ $k M) / M$ in the above inclusion, which gives (22). Hence, $W$ is pseudo-Lipschitz around $\left(u_{0}, x_{0}\right)$ with constant, $l M /(1-\gamma-k M)$.

Next, we consider the case when the constraint is a closed subset. In this case, both $x \mapsto F(x, u)$ and $x \mapsto G(x, u)$ should be locally Lipschitz, but a condition made on the $\gamma-\mathrm{G}$ inverse derivative is less strict.

Theorem 3.2. Let $K \subset X$ be a closed subset and let $\Omega$ be a neighborhood of $K$. Suppose that $F, G$ satisfy (H1)-(H3) and (F3)-(F5) below.

(F3) For each $u \in B_{U}\left(u_{0}, r\right)$ and each $x \in K \cap B_{X}\left(x_{0}, \delta\right), F(x, u)+$ $G(x, u)$ has a minimum point; that is, there exists $y \in F(x, u)+$ $G(x, u)$ such that $\|y\|=d(0, F(x, u)+G(x, u))$. 
(F4) For each $u \in B_{U}\left(u_{0}, r\right), x \mapsto F(x, u)$ is $\varepsilon$ - $\delta$-usc and, when restricted to $\Omega \cap B_{X}\left(x_{0}, \delta\right)$, is Lipschitz with constant $k_{1}$.

(F5) For each $u \in B_{U}\left(u_{0}, r\right)$, the mapping $x \mapsto F(x, u)$ possesses a $\gamma$-G inverse derivative $\Gamma_{u}(x)$ at each $x \in K \cap B_{X}\left(x_{0}, \delta\right)$ and there exist $\mu \in$ $[0,1), M>0$ such that

$0 \leq \mu\left(k_{1}+k\right) M+k M+\gamma<1$,

$\left\|\Gamma_{u}(x) y\right\| \leq M\|y\|, \quad$ for $y \in B_{y}$ and $x \in K \cap B_{X}\left(x_{0}, \delta\right)$,

$\Gamma_{u}(x) B_{Y} \subset T_{K}(x)+\mu \Gamma_{u}(x) B_{Y}, \quad$ for $x \in K \cap B_{X}\left(x_{0}, \delta\right)$,

where $T_{K}(x):=\left\{v \in X: \liminf _{h \rightarrow 0} h^{-1} d(x+h v, K)=0\right\}$ is the tangent cone of $K$ at $x$.

Let

$$
\begin{aligned}
\eta= & \sup \left\{r_{1} \leq r: \sup _{u \in B_{U}\left(u_{0}, r_{1}\right)} d\left(0, F\left(x_{0}, u\right)+G\left(x_{0}, u\right)\right)\right. \\
& \left.<\left[\frac{\left[1-\gamma-\mu\left(k_{1}+k\right) M-k M\right]}{(1+\mu) M}\right] \delta\right\} .
\end{aligned}
$$

Then, for each $u \in B_{U}\left(u_{0}, \eta\right)$, there exists $x_{u} \in K \cap B_{X}\left(x_{0}, \delta\right)$ such that

$$
0 \in F\left(x_{u}, u\right)+G\left(x_{u}, u\right) \text { and } \lim _{u \rightarrow u_{0}} x_{u}=x_{0} .
$$

In addition, if $u \mapsto F(x, u)+G(x, u)$ is locally Lipschitz at $u_{0}$ with constant $l$ uniformly in $x \in K \cap B_{X}\left(x_{0}, \delta\right)$, then the constrained implicit mapping $W$ defined in Theorem 3.1 is pseudo-Lipschitz with constant $l(1+\mu) M /\left[1-\mu\left(k_{1}+k\right) M-\right.$ $k M-\gamma]$.

Proof. Let $u \in B_{U}\left(u_{0}, \eta\right)$. Then, by our assumptions, there exists $\varepsilon_{0} \in$ $(0,(1-\gamma-k M) / M)$ such that

$$
d\left(0, F\left(x_{0}, u\right)+G\left(x_{0}, u\right)\right)<\varepsilon_{0} \delta .
$$

Let $\varepsilon \in\left(\varepsilon_{0},\left(1-\mu\left(k_{1}+k\right) M-k M-\gamma\right) /(1+\mu) M\right)$. Similarly to the proof of Theorem 3.1, by applying the Ekeland variational principle to the function

$$
V(x):=d(0, F(x, u)+G(x, u))
$$

in the metric space $K$, we see that there exists $x_{u} \in K$ such that

$$
\begin{aligned}
& V\left(x_{u}\right) \leq V(x)\|+\varepsilon\| x-x_{u} \|, \quad \text { for all } x \in K, \\
& \left\|x_{u}-x_{0}\right\| \leq(1 / \varepsilon) V\left(x_{0}\right) \leq(1 / \varepsilon) \varepsilon_{0} \delta<\delta .
\end{aligned}
$$


Suppose that $0 \notin F\left(x_{u}, u\right)+G\left(x_{u}, u\right)$. By assumption (F3), there exists $y \in$ $F\left(x_{u}, u\right)+G\left(x_{u}, u\right), y \notin 0$, such that $\|y\|=V\left(x_{u}\right)$. Let

$$
z=\Gamma_{u}\left(x_{u}\right)(-y / \lambda\|y\|), \text { with } \lambda>1 \text {. }
$$

Then, our assumptions (24) and (27) imply that there exists $y \in B_{Y}$ By such that $z \in T_{K}\left(x_{u}\right)+\mu \Gamma_{u}\left(x_{u}\right) y$. By Proposition 4.1 of Ref. 12, there exist $v_{n} \in Y, h_{n} \rightarrow 0$ such that

$$
u_{n} \rightarrow z-\mu \Gamma_{u}\left(x_{u}\right) y, \quad x_{u}+h_{n} v_{n} \in K .
$$

Clearly, $\{z\} \cup\left\{v_{n}\right\}$ is bounded. We may suppose that

$$
x_{u}+h_{n} z, x_{u}+h_{n} v_{n} \in \Omega \cap B_{X}\left(x_{0}, \delta\right) .
$$

Substituting $x=x_{u}+h_{n} v_{n}$ into (26) and using our Lipschitz assumptions, we obtain

$$
\begin{aligned}
V\left(x_{u}\right) \leq & V\left(x_{u}\right)+\varepsilon h_{n}\left\|v_{n}\right\| \\
\leq & d\left(0, F\left(x_{u}+h_{n} z, u\right)+G\left(x_{u}, u\right)\right)+H_{Y}\left(G\left(x_{u}+h_{n} v_{n}, u\right), G\left(x_{u}, u\right)\right) \\
& +H_{Y}\left(F\left(x_{u}+h_{n} z, u\right), F\left(x_{u}+h_{n} v_{n}, u\right)\right)+\varepsilon h_{n}\left\|v_{n}\right\| \\
\leq & d\left(0, F\left(x_{u}+h_{n} z, u\right)+G\left(x_{u}, u\right)+h_{n}(\gamma / \lambda) \bar{B}_{Y}\right) \\
& +h_{n} \gamma / \lambda+k_{1} h_{n}\left\|z-v_{n}\right\|+(k+\varepsilon) h_{n}\left\|v_{n}\right\| .
\end{aligned}
$$

By the definition of $\gamma-\mathrm{G}$ inverse derivative and using the same method as used in Theorem 3.1 (with $\beta=\theta=0$ ), we have

$$
V\left(x_{u}\right) \leq V\left(x_{u}\right)-h_{n} / \lambda+h_{n} \gamma+k_{1} h_{n}\left\|z-v_{n}\right\|+(k+\varepsilon) h_{n}\left\|v_{n}\right\|+\left\|o\left(h_{n}\right)\right\|,
$$

for each sufficiently large $n$. This shows that

$$
1 / \lambda \leq k_{1}\left\|z-v_{n}\right\|+(k+\varepsilon)\left\|v_{n}\right\|+\gamma+\left\|o\left(h_{n}\right)\right\| / h_{n} .
$$

By letting $\lambda \rightarrow 1, n \rightarrow \infty$, we obtain

$$
\begin{aligned}
1 & \leq k_{1}\left\|\mu \Gamma_{u}\left(x_{u}\right) y\right\|+\gamma+(k+\varepsilon)\left\|z-\mu \Gamma_{u}\left(x_{u}\right) y\right\| \\
& \leq \mu k_{1} M\|y\|+\gamma+(k+\varepsilon)\|z\|+(k+\varepsilon) \mu M\|y\| \\
& \leq \mu\left(k_{1}+k\right) M+k M+\varepsilon(1+\mu) M+\gamma<1 .
\end{aligned}
$$

This is a contradiction. Therefore,

$$
0 \in F\left(x_{u}, u\right)+G\left(x_{u}, u\right) .
$$

The remainder of the proof is the same as that in Theorem 3.1.

If the constraint $K$ in Theorem 3.2 is convex, then

$$
\Gamma_{u}(x) B_{Y} \subset \overline{T_{K}(x)}=T_{K}(x) ;
$$


that is, (24) holds also with $\mu=0$. So, in this case, we can choose $\mu$ in (24) arbitrarily and the definition of $\eta$ could be replaced by $\eta=0$.

Using the above theorems and the sufficient conditions obtained in Section 2, some concise implicit function or open-mapping theorems can be obtained. In Particular, if we apply Proposition 2.2, the corollary will be a generalization to the corresponding result in Ref. 7.

To close the paper, we give a theorem in the case where $X$ is finite dimensional and both $F, G$ are single-valued. For the proof, we need the following proposition which is indicated by Theorem 2.1 in Ref. 13, although $Y=\mathbb{R}$ in Ref. 13; therefore, its proof is omitted.

Proposition 3.1. See Ref. 13 Suppose that $f: \mathbb{R}^{n} \rightarrow Y$ is locally Lipschitz and Gâteaux differentiable at $x_{0}$. Then, for each $\varepsilon>0$, there exists $\delta>0$ such that

$$
\left\|f\left(x_{0}+v\right)-f\left(x_{0}\right)-\mathrm{D} f\left(x_{0}\right) v\right\| \leq \varepsilon\|v\|, \quad \text { for all } v \in \mathbb{R}^{n} \text { with }\|v\| \leq \delta .
$$

Theorem 3.3. Suppose that $f, g: \mathbb{R}^{n} \times U \rightarrow Y$ are continuous functions and satisfy the following conditions:

(i) $x \mapsto f\left(x, u_{0}\right)$ is locally Lipschitz, Fréchet differentiable at $x_{0}$ with derivative $\mathrm{D}_{1} f\left(x_{0}, u_{0}\right)$.

(ii) $f(x, u)+g(x, u) \in$ range $\left(\mathrm{D}_{1} f\left(x_{0}, u_{0}\right)\right)$ for all $(x, u)$ in a neighborhood $\mathcal{W}$ of $\left(x_{0}, u_{0}\right)$.

(iii) $v \mapsto \mathrm{D}_{1} f\left(x_{0}, u_{0}\right) u$ is Lipschitz invertible and the Lipschitz constant of the inverse is $\mathrm{M}$.

(iv) There exist $k, k_{1}, \geq 0$, such that $k M<1$, such that

$$
\begin{aligned}
\left\|g(x, u)-g\left(x_{0}, u_{0}\right)\right\| & \leq k\left\|x-x_{0}\right\| \\
& +k_{1}\left\|u-u_{0}\right\|, \quad \text { for } \operatorname{all}(x, u) \in \mathcal{W} .
\end{aligned}
$$

Then, for each neighborhood $\mathcal{V}$ of $x_{0}$, there exists a neighborhood $\mathcal{U}$ of $u_{0}$ and a function $\phi: \mathcal{U} \rightarrow \mathcal{V}$ such that

$$
f(\phi(u), u)+g(\phi(u), u)=f\left(x_{0}, u_{0}\right)+g\left(x_{0}, u_{0}\right), \quad \text { for every } u \in \mathcal{U} .
$$

Proof. Without loss of generality, let

$f\left(x_{0}, u_{0}\right)=g\left(x_{0}, u_{0}\right)=0$.

Let

$\eta=1-k M \quad$ and $\quad P=\mathrm{D}_{1} f\left(x_{0}, u_{0}\right)$. 
From Proposition 3.1 and our assumptions, it follows that there exists $\alpha>0$ such that

$x_{0}+\alpha \bar{B} \subset \mathcal{V} \quad$ and $\quad\left\|f\left(x_{0}+x, u_{0}\right)-P x\right\| \leq(\eta / 3 M) \alpha, \quad$ for $x \in \alpha \bar{B}$.

The compactness of $\alpha \bar{B}$ implies that there exists $\beta \in\left(0, \eta \alpha / 6 M k_{1}\right)$ such that $\left\|f\left(x_{0}+x, u\right)-f\left(x_{0}+x, u_{0}\right)\right\| \leq(\eta / 3 M) \alpha, \quad$ for all $x \in \alpha \bar{B}, u \in B_{U}\left(u_{0}, \beta\right)$.

By our assumption (iv), for any $x \in \alpha \bar{B}, u \in B_{U}\left(u_{0}, \beta\right)$, we have

$$
\left\|g\left(x_{0}+x, u\right)\right\|=\left\|g\left(x_{0}+x, u\right)-g\left(x_{0}, u_{0}\right)\right\| \leq k \alpha+k_{1} \beta .
$$

Now, let $u \in B_{U}\left(u_{0}, \beta\right)$ be given and let

$$
N_{u}(x)=x-P^{-1}\left(f\left(x_{0}+x, u\right)+g\left(x_{0}+x, u\right)\right), \quad \text { for each } x \in \alpha \bar{B} .
$$

By the Lipschitz property of $P^{-1}$ and our assumption (ii), we see that $N_{u}$ is a well-defined continuous operator on $\mathbb{R}^{n}$. (29)-(31) imply that, for each $x \in \alpha \bar{B}$, we have

$$
\begin{aligned}
\left\|N_{u}(x)\right\| \leq & \left\|P^{-1} P x-P^{-1}\left(f\left(x_{0}+x, u_{0}\right)+g\left(x_{0}+x, u_{0}\right)\right)\right\| \\
& +\| P^{-1}\left(f\left(x_{0}+x, u_{0}\right)+g\left(x_{0}+x, u_{0}\right)\right)-P^{-1}\left(f\left(x_{0}+x, u\right)\right. \\
& \left.+g\left(x_{0}+x, u\right)\right) \| \\
\leq & \left\|P^{-1} P x-P^{-1} f\left(x_{0}+x, u_{0}\right)\right\| \\
& +\left\|P^{-1} f\left(x_{0}+x, u_{0}\right)-P^{-1}\left(f\left(x_{0}+x, u_{0}\right)+g\left(x_{0}+x, u_{0}\right)\right)\right\| \\
& +M\left\|f\left(x_{0}+x, u_{0}\right)+g\left(x_{0}+x, u_{0}\right)-f\left(x_{0}+x, u\right)-g\left(x_{0}+x, u\right)\right\| \\
\leq & M \| P x-f\left(x_{0}+x, u_{0}\|+M\| g\left(x_{0}+x, u\right) \|\right. \\
& +M\left\|f\left(x_{0}+x, u_{0}\right)-f\left(x_{0}+x, u\right)\right\|+M \| g\left(x_{0}+x, u_{0}\right) \\
& -g\left(x_{0}+x, u\right) \| \\
\leq & M(\eta / 3 M) \alpha+M k \alpha+M k_{1} \beta+M(\eta / 3 M) \alpha+M k_{1} \beta \\
\leq & \alpha(\eta+k M) \\
= & \alpha .
\end{aligned}
$$

That is $N_{u}$ maps $\bar{B}$ into itself. So, $N_{u}$ has fixed point $x_{u} \in \bar{B}$; therefore,

$$
P^{-1}\left(f\left(x_{0}+x_{u}, u\right)+g\left(x_{0}+x_{u}, u\right)\right)=0 .
$$

By letting

$$
\phi(u)=x_{0}+x_{u},
$$

we obtain

$$
f(\phi(u), u)+g(\phi(u), u)=P(0)=0 .
$$

This completes the proof. 
Remark 3.1. If $g(x, u) \equiv 0$ and $U=\mathbb{R}^{m}, Y=\mathbb{R}^{n}$, we obtain the main result of Ref. 3. We remark that, in Ref. 3, an extra condition such as (ii) should be imposed to ensure that the operator $N_{u}$ is well defined. We notice also that the Gateaux differentiability and the Lipschitz requirement for the derivative, as made in Ref. 3, imply the Frechet differentiability.

\section{References}

1. BIAN W., and WeBB, J. R. L., Constrained Open-Mapping Theorems and Applications, Journal of the London Mathematical Society, Vol. 60, pp. 897-911, 1999.

2. DONTCHEV, A. L., Implicit Function Theorems for Generalized Equations, Mathematical Programming, Vol. 70, pp. 91-106, 1995.

3. KUNTZ, K., An Implicit Function Theorem for Directionally Differentiable Functions, Journal of Optimization Theory and Applications, Vol. 86, pp. 263-270, 1995.

4. RAY, W. O., and CRAMER, W. J., Solvability of Nonlinear Operator Equations, Pacific Journal of Mathematics, Vol. 95, pp. 37-50, 1981.

5. RAY, W. O., and WALKER, A. M., Mapping Theorems for Gâteaux Differentiable and Accretive Operators, Nonlinear Analysis: Theory, Methods, and Applications, Vol. 6, pp. 423-433, 1982.

6. Milojević, P. S., Implicit Function Theorems, Approximate Solvability of Nonlinear Equations, and Error Estimates, Journal of Mathematical Analysis and Applications, Vol. 211, pp. 424-459, 1997.

7. PÁLES, Z., Inverse and Implicit Function Theorems for Nonsmooth Maps in Banach Spaces, Journal of Mathematical Analysis and Applications, Vol. 209, pp. 202-220, 1997.

8. FrankowsKa, H., An Open-Mapping Principle for Set-Valued Maps, Journal of Mathematical Analysis and Applications, Vol. 127, pp. 172-180, 1987.

9. WELSH, S. C., Open Mapping and Solvability of Nonlinear Equations in Banach Spaces, Proceedings of Section A: Mathematics, Royal Society of Edinburgh, Vol. 126, pp. 239246, 1996.

10. DeIMLING, K., Nonlinear Functional Analysis, Springer Verlag, Berlin, Germany, 1985.

11. Aubin, J. P., and Frankowska, H., Set-Valued Analysis, Birkhäuser, Boston, Massachusetts, 1990.

12. DeIMLING, K., Multivalued Differential Equations, Walter de Gruyter, Berlin, Germany, 1992.

13. PallaschKe, D., Recht, P., and Urbański, R., On Locally-Lipschitz Quasidifferentiable Functions in Banach Spaces, Optimization, Vol. 17, pp. 287-295, 1986. 